\title{
A Finite Element Model to Predict the Effect of Porosity on Elastic Modulus in Low Porosity Materials
}

\author{
Liam Morrissey \\ Department of Mechanical Engineering \\ Memorial University of Newfoundland \\ St. John's, Canada
}

\author{
Sam Nakhla \\ Department of Mechanical Engineering \\ Memorial University of Newfoundland \\ St. John's, Canada
}

\begin{abstract}
I. Abstract - The effect of porosity on elastic modulus in low porosity materials is investigated. First, several models used to predict the reduction in elastic modulus due to porosity are compared with a compilation of experimental data to determine their ranges of validity and accuracy. The overlapping solid spheres model is found to be most accurate with the experimental data and valid between $3-10 \%$ porosity. Next, a FEM is developed with the objective of demonstrating that a macroscale plate with a center hole can be used to model the effect of microscale porosity on elastic modulus. The FEM agrees best with the overlapping solid spheres model and shows higher accuracy with experimental data than the overlapping solid spheres model.
\end{abstract}

\section{Keywords-porosity, elastic modulus, FEA}

\section{INTRODUCTION}

\section{A. Background}

Porosity can cause changes to a material's behaviour under loading that is different than predicted. In the extreme case, these changes, if unaccounted for, could lead to major safety and economic concerns. Therefore, the ability to accurately and easily model the effect of porosity is of paramount importance during the design and testing stages. While metals are typically not intended to contain significant pores, micro pores can be present due to manufacturing defects. These pores, which at first glance may appear inconspicuous, can increase in size during service in harsh environmental conditions. For example, in oil and gas pipelines and in hydrogen fuel tanks (typically found in aerospace), materials can be subject to high concentrations of hydrogen. Over time, hydrogen can diffuse into the material and recombine into $\mathrm{H}_{2}$, increasing the size of the initial voids and thus raising the porosity to a level that may effect the elastic modulus of the material [1-2]. However, while the effect of low levels of porosity on the yield stress has been comprehensively studied and captured in finite element software, the effect on the elastic modulus requires further research [3].

\section{B. Porosity and its Effect on Modulus}

Significant research has been completed on the relationship between porosity and elastic modulus. Models typically agree that the relationship falls into categories based on the level of porosity. For example, the work of Zhang and Wang in [4] has recommend subdividing porosity levels into low, medium and high porosity. Where low porosity is less than $10 \%$, medium porosity is $10-70 \%$ and, finally, high porosity is greater than $70 \%$. Therefore the approach taken to predict the effect of porosity on elastic modulus depends first on the degree of porosity in the material. It is important to remember that the numbers in these ranges are not necessarily exact and could possibly be better described as extremely low porosity, extremely high porosity, and then the large middle level [5]. In low porosity materials $(<10 \%$ porosity) there are several suggested relationships between porosity and elastic modulus that depend only on porosity. However, they do not predict the same influence of porosity and create a potential confusion when attempting to model a porous material. Therefore, this study attempted to clarify this confusion by evaluating these models and then demonstrating the applicability of a finite element model (FEM) to accurately predict the change in elastic modulus due to porosity.

1) Low Porosity Materials

For materials of low porosity there have been both analytical and experimental studies that developed a relationship to predict the elastic modulus in terms of the porosity. In this porosity level the elastic modulus is typically determined based on the assumption that voids are not interacting and that void fraction, and not void orientation, effects the materials behaviour [6]. As a result, models in the low porosity level are independent of the material and depend only on the degree of porosity.

For example, it has been suggested that a more simplified approach to modelling the elastic modulus of a porous material can be to consider the rule of mixtures used in composites to relate the modulus of two phases based on a volumetric fraction. In this case, one of the phases is considered as the solid non porous material and the other phase is the empty voids $[5,7]$. However, there is a lack of research on the validity of this model or its ranges of accuracy as compared to experimental data. As another example, Coble and Kingery in [8] developed a model to describe materials with spherical pores through testing of cast alumina with porosity from 5$50 \%$. Elastic modulus was then measured using samples in transverse bending and a model was generated that best fit experimental findings. Similarly, Maiti et al. in [9] developed a relationship between modulus and porosity by measuring the mechanical properties of three types of cellular solids as a 
function of density. An analytical model was then developed based on the concept that the loading of foam first causes deflection in the cells walls, which can be calculated by relating the densities of the foams to the cell walls. While this study was initially developed to model properties as a function of density, the ratio of the densities can also be related to a porosity ratio; allowing for the subsequent development of a model to predict the behaviour of porous materials. Finally, in [10] $\mathrm{Lu}$ et al. studied the effect of porosity on common industrial materials such as carbon rods, woods, ceramics and foams. Based on these experimental results a micromechanical theoretical model was then developed that related the elastic modulus to the porosity for porosity less than $30 \%$. This model considered the material as an isotropic matrix with $n$ spherical voids of varying sizes. Average shear strains were then calculated for a given shear stress, allowing the shear and elastic modulus to be calculated as a function of porosity. The theoretical model showed good agreement with the experimental data presented.

\section{2) Intermediate Porosity}

It is important to note that some models developed for the intermediate porosity level have also shown accuracy with experimental values in the low porosity level. As a result, some of these analytical models were also considered for their accuracy in predicting the elastic modulus in low porosity materials. In medium porosity materials pores can interact and lead to stress redistribution in the material. Moreover, there exists a critical porosity fraction that, when reached, the material loses all stiffness and load carrying capability. Accounting for this critical porosity value, data Bert et. al. in [11] presented (1) to predict the elastic modulus of medium porosity materials through an empirical observation of trends in experimental data. This model was developed based on the assumption that the ratio of porous material property to the solid material property is proportional to the ratio of minimum solid contact area to total cross-section area in the porous material [12]. The parameters $\phi_{0}$ and $\mathbf{n}$ must be determined for each model/material by best fitting to experimental/analytical results.

$$
\mathrm{E}(\phi)=\mathrm{E}_{0}\left(1-\left(\phi / \phi_{0}\right)\right)^{\mathrm{n}}
$$

Where $E_{0}$ is the elastic modulus of the material without pores, $\phi_{0}$ is the critical porosity value for the given material (between .37 and .97), $\phi$ is the porosity of the material, and $n$ is a power exponent that must be determined for each model/material.

Building on this model, in [13] Roberts and Garboczi used finite element analysis to study the influence of porosity and pore shape on the elastic properties of model porous ceramics. The study developed three different FEMs by placing spheres/ellipses in a unit cell to create various pore shapes and then best fitting the results to the model suggested by Bert in [5] to determine the $\phi_{0}$ and $n$ parameters. Predicted elastic moduli agreed well with experimental data and several models also accurately predicted the elastic modulus of materials with porosity below $10 \%$. Therefore these models will also be considered as potential models for the low porosity level.

Similarly, Hardin and Beckermann in [5] used a FEM to determine the best fit parameters for cast steel. First, a simple tension test and radiographic measurement was performed and a FE mesh was mapped onto an object in Abaqus. However, due to the microscopic nature of the pores, a small representative volume with a defined porosity fraction was used. A relationship for porosity was then determined by minimizing the difference between the FEM and experimental values and best fitting the results to the model suggested by Bert in [5].Results demonstrated that this method was accurate in the prediction of strains but had significant error in predicting the elastic modulus. The paper concluded that there was no correlation between average porosity and elastic modulus and that the reason for this error in modulus was because the FEM was a representative sample of the total porosity [5]. However, while the results may not be as useful in the prediction of elastic modulus, they serve to shed light on the importance of a FEM fully accounting for variations in porosity in the material.

\section{Finite Element Models}

Perhaps the most commonly referenced FEM for porous metals is the porous plasticity model found in Abaqus [14]. This model is based on the Gurson-Needleman-Tvergaard (GTN) model originally developed by Gurson in [15] and later extended by Tvergaard in [3] for the failure of ductile materials due to void coalescence. This model defines a yield potential based on an isolated spherical void and forms the basis of the porous plasticity model currently used in Abaqus to predict the behaviour of mildly voided materials. However, while the model is able to accurately predict micromechanical parameters such as yield stress, it is unable to account for the macro scale effects of the micro scale pores. For example, Hardin and Beckermann in [16] investigated using the porous plasticity model to model the influence of porosity on the yielding of cast steel. As porosity was changed the only observable difference in the stress strain curves was a change in yield stress [16]. Therefore, because there was no change in the slope of linear portion of the plot, the model does not predict a change in elastic modulus due to porosity. This finding serves to highlight the confusion at hand. While studies and intuition dictate that porosity should effect the modulus, the most commonly used models appear to ignore or not account for the effect on elastic modulus.

\section{Purpose of Study}

As can be seen from the above review, several models exist to predict the influence of porosity on elastic modulus. However, because there is no study on the ranges of validity and relative accuracy of these models there is a potential confusion when attempting to predict the elastic modulus of a low porosity material. Moreover, the GTN model, a popular microscale model in Abaqus for low porosity materials, only adds to the confusion by predicting a change in the yield stress and no change in the elastic modulus. As a result, Abaqus does not have the capability to accurately predict the elastic modulus of a porous material. To help eliminate this gap in knowledge this study focused on the evaluation of models used for materials in the low porosity level. The first objective of this study was to compare the present models and to clearly identify their validity when compared to experimental data. Next, a FEM was developed with the objective of demonstrating that a 
macroscale plate with a center hole can be used to the effect of microscale porosity on elastic modulus.

\section{PROCEDURES}

\section{A. Evaluation of Existing Models}

The first step in this study was to examine experimental data to verify that that there was indeed an effect of porosity on elastic modulus. This was achieved by compiling a set of previously conducted experimental data on the elastic modulus of materials with porosity ranging from $0-10 \%$. Next, this experimental data was used to evaluate the previously suggested relationships between porosity and elastic modulus to determine their relative accuracy.

\section{B. Finite Element Model Study}

After determining which relationship best predicted the effect of porosity on elastic modulus a simplified FEM was then developed using the finite element software Abaqus. First, a large 2D stainless steel plate in plane stress was developed and a pressure load was applied on its right edge. The plate was fixed in the U1 direction on its left edge to model a tension test. A simulation was then run to produce a stress strain curve using the applied pressure and change in length of the plate. Finally, the slope of this curve was used to obtain an effective elastic modulus of $200 \mathrm{GPa}$; matching the inputted value and therefore verifying the model.

Next, python scripting was used to investigate the effect of pore orientations on elastic modulus. The pores simulated various degrees of porosity in the material. The above test was replicated for a range of pore orientations and stress strain curves were used to calculate the elastic modulus at 2,4,6 8 and $10 \%$ porosity. A summary of the pore orientations tested is shown in Figure 1.

Finally, based on the findings of pore orientation, python scripting was used to test plates with a center pore accounting for $1-10 \%$ of the total surface area of the plate. The center pore simulated various degrees of porosity in the plate based on the assumption that total pore volume, and not pore orientation, effects the modulus for low porosity materials. The slope of the stress strain curve was then used to obtain the effective elastic modulus for the steel at various degrees of porosity. These results were then compared with experimental data and the most accurate models from above to demonstrate that a macroscale FEM accurately predicted the influence of porosity on elastic modulus.

\section{RESULTS AND DisCUSSION}

\section{A. Effect of Porosity on Elastic Modulus}

While several models exist to predict the change in elastic modulus due to porosity, their relative accuracy and ranges of validity remains unknown. To close this knowledge gap a comparison between several models and experimental data was conducted. Figure 3 compiles a set of experimental data on the percent reduction in elastic modulus for a range of porous materials. The data observed a linear trend of increasing percent reduction in elastic modulus with increasing porosity $\left(\mathrm{R}^{2}=.77\right)$. Therefore, it is clear that porosity has a direct effect on the elastic modulus of a material. The wide range of materials and preparation techniques for the experimental data also shed light upon the variables that should be considered when modelling porosity. For example, alumina values were obtained from two different sources [17-18]. In [18], Asmani et. al shaped alumina pellets via uniaxial pressing and then sintered these pellets at various temperatures to produce closed porosity shapes. The elastic modulus was then measured using a pulse echo method that calculated the transit time and longitudinal/transverse wave velocities. In contrast, the data provided by Knudsen in [17] was a compilation for a range of tests on the elastic modulus of alumina. Samples were prepared via cold and hot pressing and elastic modulus was determined both statically and dynamically. Therefore, given that both data sets closely follow the same trend, it appears that preparation/measuring techniques do not influence the reduction in elastic modulus. While the majority of materials closely followed this trend, porosity in $\mathrm{HfO}_{2}$ resulted in a lower reduction in elastic modulus than other materials. The $\mathrm{HfO}_{2}$ samples were prepared using a dried powder that was formed calcined, and then stabilized with approximately $30 \% \quad \mathrm{Er}_{2} \mathrm{O}_{3}$ [19]. However, this additive was shown to change the microstructure and effect the baseline elastic modulus [19]. As a result, the stabilizer may have changed the porosity of the material and lead to less of a reduction in elastic modulus. Moreover, when the data for $\mathrm{HfO}_{2}$ is ignored the linearity of the experimental values raises to and R-squared value of .87 . Overall, because the data for several different materials followed the same linear trend it supports the conclusion that models for low porosity materials do not need to be material specific and that the pore volume is critical parameter.

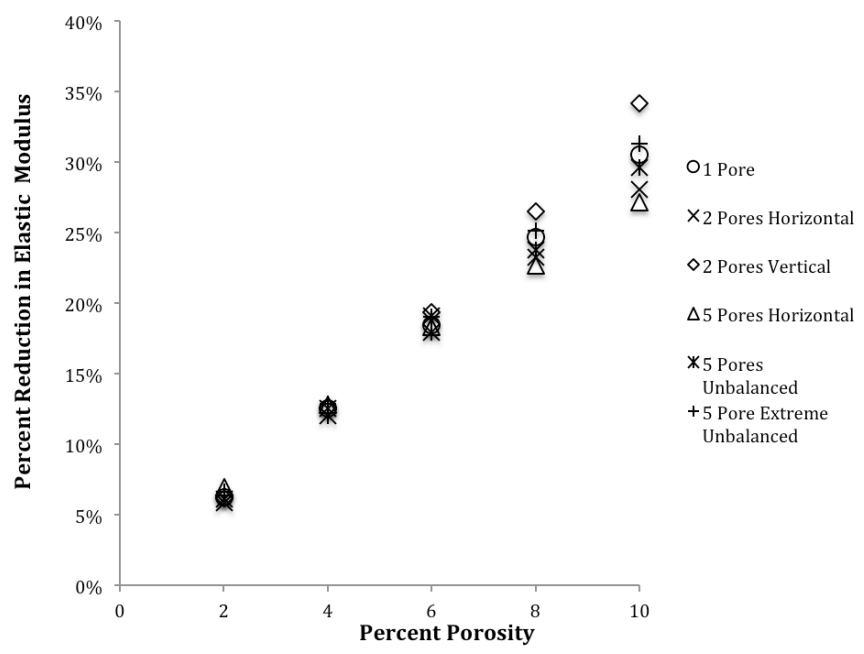

Fig. 1 - A compilation of experimental data for the percent reduction in elastic for various materials with porosity between 1-10\% (Alumina 1 [17], Alumina 2[18], thermoset polyester resin [20], $\mathrm{HfO}_{2}$ [19], sintered Iron [21], $\mathrm{MgAl}_{2} \mathrm{O}_{4}$ ([22])

\section{B. Evaluation of Existing Models}

Next, 6 models for low porosity materials were evaluated against the experimental data above. First, the three 
relationships modelled by Roberts and Garboczi in[13] based on various pore orientations were considered (2-4). These equations were developed by placing either spheres or ellipses into a unit cell such that the orientations of these pores created various pore shapes. Results were then best fit to (1) to determine the $\phi_{0}$ and 12 parameters.

$$
\begin{aligned}
& \mathrm{E}(\phi)=\mathrm{E}_{0}(1-(\phi / 0.652))^{2.23} \\
& \mathrm{E}(\phi)=\mathrm{E}_{0}(1-(\phi / 0.818))^{1.65} \\
& \mathrm{E}(\phi)=\mathrm{E}_{0}(1-(\phi / 0.798))^{2.23}
\end{aligned}
$$

Where (2) was developed for overlapping solid spheres by placing solid spheres at random points in a unit cell, (3) was developed for overlapping spherical pores by interchanging the roles of the solid and pore phase of the overlapping solid sphere model and (4) was developed for overlapping ellipsoidal pores by changing to spherical pores to overlapping oblate ellipsoidal pores bound by a surface.

In addition to these models, the rule of mixtures (5) in [7], the model for materials with spherical holes developed by Coble and Kingery (6) in [8], the model for closed cell porous materials developed by Maiti et al. (7) in [9], and the micromechanical model suggested by Lu et al. (8) in [10] were also considered.

$$
\begin{aligned}
& \mathrm{E}(\phi)=\mathrm{E}_{0}(1-\phi) \\
& \mathrm{E}(\phi)=\mathrm{E}_{0}\left(1-1.86 \phi+0.86 \phi^{2}\right) \\
& \mathrm{E}(\phi)=\mathrm{E}_{0}(1-(\phi))^{3} \\
& \mathrm{E}(\phi)=\mathrm{E}_{0}(1-2 \phi)\left(1+4 \phi^{2}\right)
\end{aligned}
$$

Figure 4 presents the predicted percent reduction in elastic modulus for each model as compared to the experimental data for low porosity materials. First from an overall perspective, all analytical models considered captured the trend of increasing percent reduction in elastic modulus with increasing porosity demonstrated by the experimental values. Between $0-2 \%$ porosity (Figure $2 \mathrm{~A}$ ) it is difficult to discern a clear trend and the range of predicted values was closely clustered between a $2-6 \%$ reduction in elastic modulus. However, as the porosity increased (Figure $2 \mathrm{~B}$ ) the range of predicted percent reduction grew to over $20 \%$ and there were stark differences in the models.

First, for the FEMs presented by Roberts and Garboczi in [13] it can be seen that the overlapping solid spheres model and ellipsoidal pores model both fell within the range of experimental values and were more accurate than the spherical pores model. Moreover, when compared to line of best fit of the experimental data the overlapping solid spheres model was the most accurate model tested. However, the micromechanical geometries of these models create potential limitations to the respective ranges of validity. For example, in the overlapping spherical pores model pores only become macroscopically connected at porosities above $30 \%$. Therefore, an overlapping solid spheres microstructure is not applicable for low porosity materials, potentially explaining the inaccuracy with the experimental. Similarly, pores in the overlapping ellipsoidal pores model only become macroscopically connected at porosities above $20 \%$. However, while the range of validity for this model was also outside the porosity level in question, it's validity was closer to the $0-10 \%$ level than the overlapping solid spheres model. This may help explain why it followed the experimental line of best fit closer than the overlapping solid spheres model. Finally, the overlapping spherical pores model was the most accurate of the three and, unlike the other models, has a microstructure than becomes macroscopically connected at porosities above $3 \%$. Therefore, this model is valid within the majority of the low porosity range and was most accurate when compared to the experimental line of best fit.

When examining the other models considered it can clearly be seen that (5), the rule of mixtures model, drastically under predicted the percent reduction in elastic modulus and did not agree with any experimental data. Similarly, the models suggested by Coble and Kingery (6) and Lu (8) both under predicted the reduction in elastic modulus and agreed only with the experimental data for $\mathrm{HfO}_{2}$ presented by Dole et al. in [19]. Therefore, these models are not recommended to predict the effect of porosity on elastic modulus. In contrast, (7), the model for closed cell porous materials developed by Maiti et al., also closely followed the experimental data and was the second most accurate model when compared to the line of best fit. This model was actually derived during the development of a model to predict mechanical properties of a cellular material in compression. The initial model predicted the elastic modulus of a foam through the ratio of the relative densities and the elastic modulus of the solid cell wall. (7) was then derived by replacing the ratio of the densities of foam to solid by one minus the porosity, and by then replacing the elastic modulus of the cell wall with the elastic modulus of the solid material.

\section{Finite Element Model}

\section{1) Effect of Pore Orientation on Elastic Modulus}

After comparing and evaluating the existing models, the next step was to examine the applicability of a FEM for low porosity materials in Abaqus. Although porosity is a microscale phenomenon, its effect can be seen on both the yield stress (a microscale property) and on elastic modulus (a macroscale property). Therefore, the fundamental question asked by this FEM was whether microscopic pores can be approximated as one bulk macroscopic hole and then be used to accurately obtain the elastic modulus, a macroscopic property. The first step to answering this question was to investigate the effect of pore orientation on elastic modulus. This was achieved by measuring the slope of the stress strain curve of a macroscopic plate in tension in Abaqus with various pore orientations accounting for $0-10 \%$ porosity. 

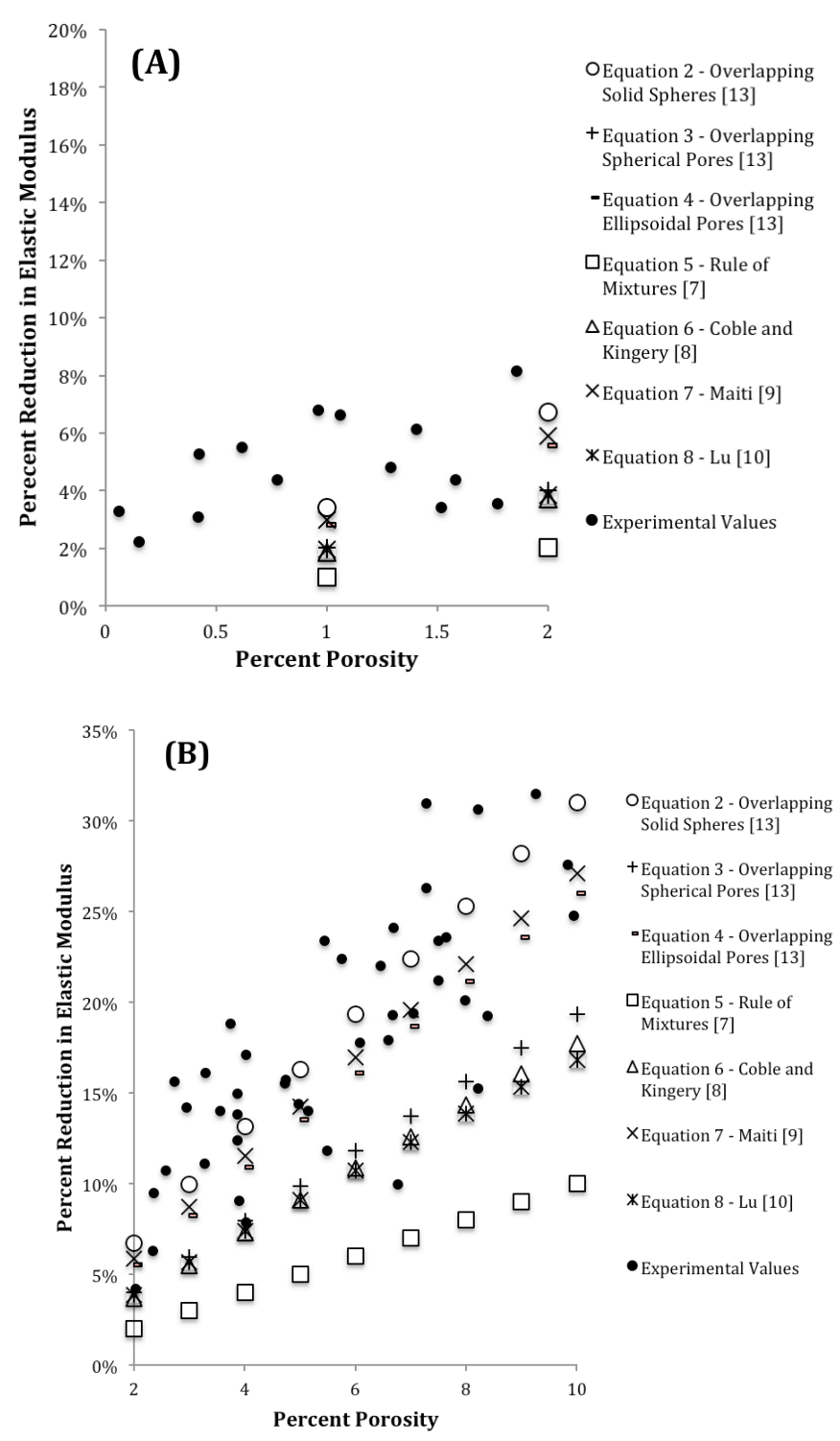

Fig. 2 - Predicted reduction in elastic modulus for various analytical models compared with experimental data for materials with porosity between $0-2 \%$ (A) and $2-10 \%$ (B)

The effect of porosity on elastic modulus for various pore orientations was compared in Figure 3. Several different pore orientations were considered that varied the size, number, and location of the pores. The maximum error between orientations occurred at $10 \%$ porosity $(8.32 \%)$, while all other errors were below $6 \%$. Therefore, changing pore orientation did not appear to cause a significant effect of the percent reduction of elastic modulus. However, it is important to note that as porosity increased the standard deviation between the various orientations also increased. For example, at $2 \%$ porosity the standard deviation between all orientations was only 0.004 and the range in predicted reduction in elastic modulus was $5.9 \%-6.9 \%$. However, as the porosities increased so did the gap in the predicted reduction of elastic modulus. For example, by simply looking at Figure 3 it is clear that data at $10 \%$ porosity the results are significantly

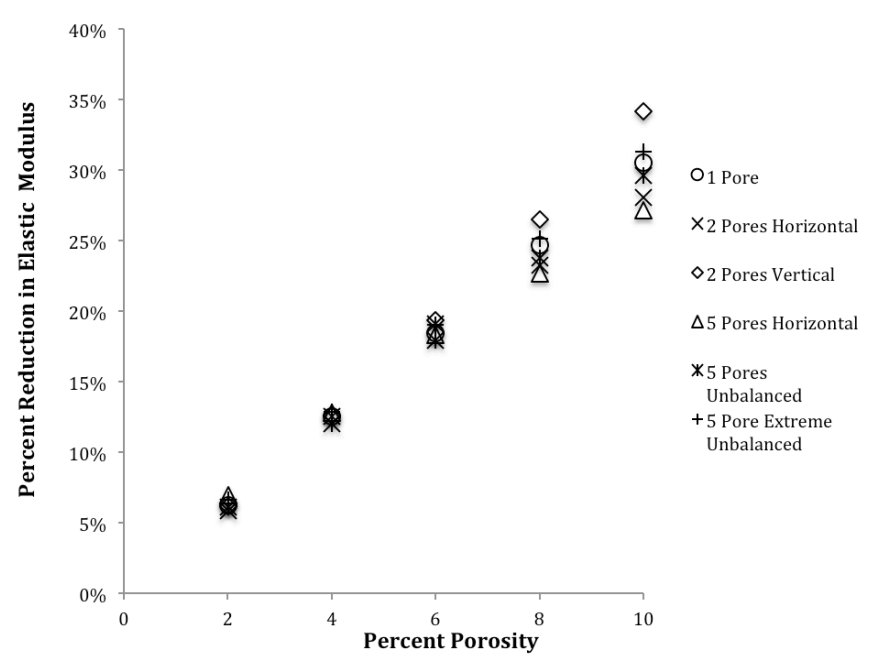

Fig. 3 - Effect of pore orientation on reduction of elastic modulus for low porosity materials.

more spread out than the at the lower porosities. At $10 \%$ porosity the standard deviation was raised by approximately seven times to 0.025 and range in values was between 27 $34 \%$. Therefore, while the assumption that pore orientation does not effect change in elastic modulus for low porosity materials was supported, there is more error in the assumption as the material nears $10 \%$ porosity. In addition, it can be seen that the 1 center pore always fell within the middle of the ranges of percent reduction in elastic modulus. Therefore, to ensure that the results were not biased by orientation, the center pore was used for comparison with analytical and experimental results.

\section{2) Comparison of Finite Element Model with Analytical} Models and Experimental Values

After investigating the effect of pore orientation in a FEM, the next step was to compare the FEM with analytical and experimental results. First, Figure 4 compares the predicted reduction in elastic modulus for porosities between $0-10 \%$ for the proposed FEM with the analytical models. The FEM produced a highly linear relationship between porosity and elastic modulus between $1-10 \%$ porosity $\left(\mathrm{R}^{2}=.9983\right)$. Over the entire porosity range the overlapping solid spheres model had the lowest average error when compared to the FEM (average error of $-4.38 \%$ with standard deviation of .0216) followed by (7) (average error of $8.67 \%$ with standard deviation of .0199). Therefore, the two models shown to be most accurate to the experimental data also matched best with the proposed FEM.

Next, Figure 5 compares the results of the FEM with experimental data from several different low porosity materials. The experimental data and FEM both closely followed a similar linear trend with the FEM falling within the range determined by the various experiments. When comparing the FEM to a linear trend line of experimental data it can be seen that the FEM followed the experimental data 
better than all other models for porosities between 5-10\% and was second only to the overlapping solid spheres model for all other porosities. Moreover, because the overlapping solid spheres model is not valid between $0-2 \%$, the FEM was both more accurate and applicable over a larger range of validity. Therefore, a macroscale plate with a hole was shown to successfully predict the elastic modulus of materials with micro pores.

\section{CONCLUSIONS}

An accurate relationship between elastic modulus and porosity is critical to safely using materials with porosity. However, while several models exist to predict the effect of porosity on elastic modulus for low porosity materials, these relationships predict varying effects of porosity. This can create confusion for engineers looking to predict the behaviour of a porous material. Therefore, this study compared commonly used models against a compilation of experimental values. While several models did not match well with experimental values, the overlapping solid spheres model (2) best agreed well with the data. Next, this paper considered a large plate with a center pore in tension to demonstrate the validity of a using a macroscale model to predict the effect of microscale pores on the elastic modulus using FEA. The FEM matched with experimental data better than the overlapping solid spheres model and, unlike this model, was applicable for the entire low porosity range. Therefore, this study successfully demonstrated the most accurate relationship between porosity and elastic modulus and then used a FEM to improve upon this model.

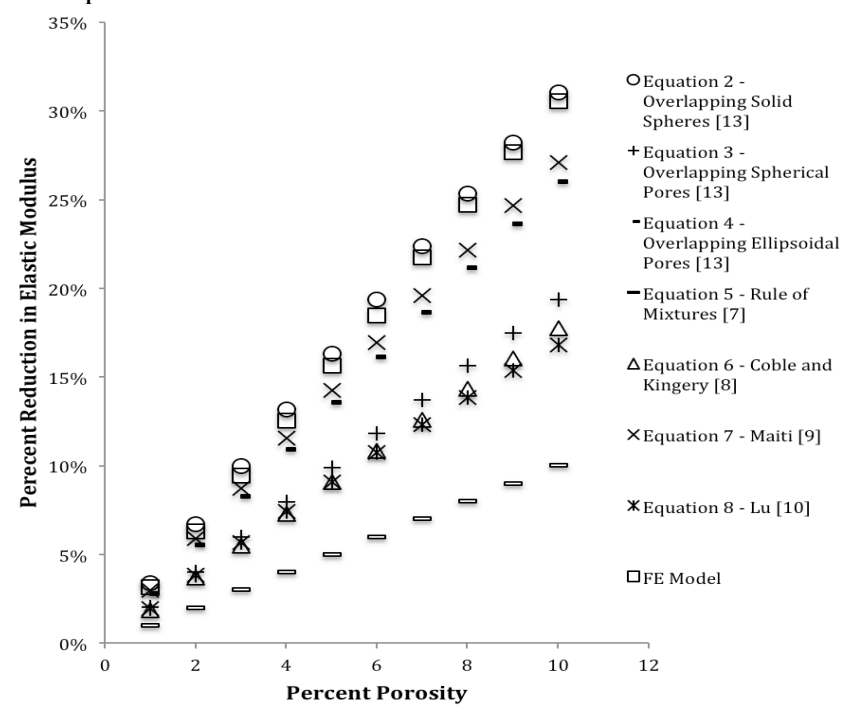

Fig. 4 - The predicted reduction in elastic modulus for porosities between $0-10 \%$ for the proposed Finite Element model as compared to analytical models.

\section{REFERENCES}

1. Y. Liang, D. C. Ahn, P. Sofronis, R. H. Dodds Jr and D. Bammann, Mechanics of Materials 2008, vol. 40, pp. 115-132. 2. M. Nagumo, Materials Science and Technology 2004, vol. 20, pp. $940-950$.

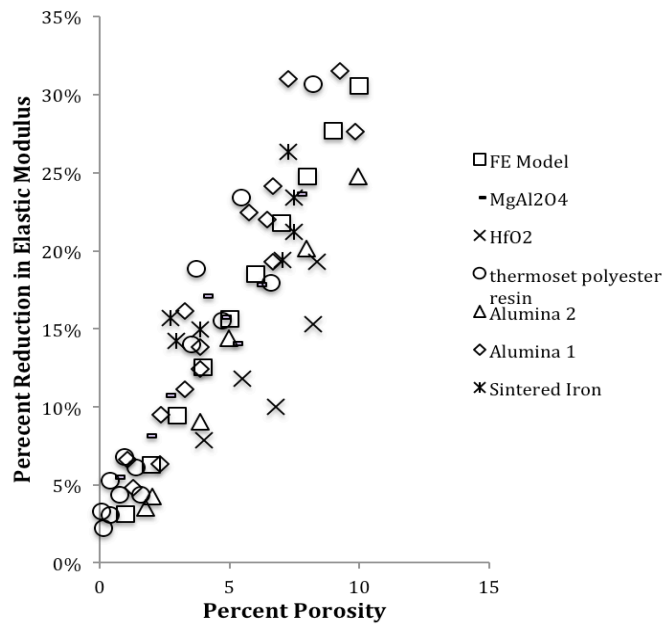

Fig. 5 - Reduction in modulus for FEM and experimental data (Alumina 1 [17], Alumina 2[18], thermoset polyester resin [20], $\mathrm{HfO}_{2}$ [19], sintered Iron [21], $\mathrm{MgAl}_{2} \mathrm{O}_{4}$ ([22]) 3 . V. Tvergaard, International Journal of Fracture 1981, vol. 17, pp. 389-407.

4. E. Zhang and B. Wang, International Journal of Mechanical Sciences 2005, vol. 47, pp. 744-756.

5. R. A. Hardin and C. Beckermann, Metallurgical and Materials Transactions A 2007, vol. 38, pp. 2992-3006. 6. J. M. Dewey, Journal of Applied Physics 1947, vol. 18, pp. 578-581.

7. T. Sumitomo, C. H. Cáceres and M. Veidt, Journal of Light Metals 2002, vol. 2, pp. 49-56.

8. R. L. Coble and W. D. Kingery, Journal of the American Ceramic Society 1956, vol. 39, pp. 377-385.

9. S. K. Maiti, L. J. Gibson and M. F. Ashby, Acta Metallurgica 1984, vol. 32, pp. 1963-1975.

10. G. Lu, G. Q. Lu and Z. M. Xiao, Journal of Porous Materials 1999, vol. 6, pp. 359-368.

11. C. W. Bert, Journal of Materials Science 1985, vol. 20, pp. 2220-2224.

12. R. W. Rice, Journal of Materials Science 2005, vol. 40, pp. 983-989.

13. A. P. Roberts and E. J. Garboczi, Journal of the American Ceramic Society 2000, vol. 83, pp. 3041-3048.

14.K. S. Hibbit, Inc.: Abaqus Theory Manual V 6.5. (2004).

15. A. L. Gurson, Journal of Engineering Materials and Technology 1977, vol. 99, pp. 2-15.

16. R. A. Hardin and C. Beckermann, In In Proceedings of the 65th SFSA Technical and Operating Conference, (2011).

17. F. P. Knudsen, Journal of the American Ceramic Society 1962, vol. 45, pp. 94-95.

18. M. Asmani, C. Kermel, A. Leriche and M. Ourak, Journal of the Eur. Ceramic Society 2001, vol. 21, pp. 1081-1086. 19. S. L. Dole, O. Hunter and F. W. Calderwood, Journal of the American Ceramic Society 1980, vol. 63, pp. 136-139. 20. K. K. Phani and R. N. Mukerjee, Journal of Materials Science 1987, vol. 22, pp. 3453-3458.

21. J. P. Panakkal, H. Willems and W. Arnold, Journal of Materials Science 1990, vol. 25, pp. 1397-1402.

22. D. F. Porter, J. S. Reed and D. L. Iii, Journal of the American Ceramic Society 1977, vol. 60, pp. 345-349. 